\title{
Extended photometric survey of near-Earth objects ${ }^{\star}$
}

\author{
S. Ieva ${ }^{1}$, E. Dotto ${ }^{1}$, E. Mazzotta Epifani ${ }^{1}$, D. Perna ${ }^{1}$, C. Fanasca ${ }^{1}$, M. Lazzarin ${ }^{2}$, I. Bertini ${ }^{3,4}$, V. Petropoulou ${ }^{5}$, \\ A. Rossi ${ }^{6}$, M. Micheli ${ }^{1,7}$, and E. Perozzi ${ }^{8}$ \\ ${ }^{1}$ INAF - Osservatorio Astronomico di Roma, Via Frascati 33, 00078 Monte Porzio Catone, Rome, Italy \\ e-mail: simone.ieva@inaf.it \\ 2 Dipartimento di Fisica e Astronomia 'Galileo Galilei', Università di Padova, Vicolo dell'Osservatorio 3, 35122 Padova, Italy \\ ${ }^{3}$ Dipartimento di Scienze e Tecnologie, Università Parthenope, Centro Direzionale, Isola C4, 80143 Naples, Italy \\ ${ }^{4}$ INAF - Istituto di Astrofisica e Planetologia Spaziali, Via del Fosso del Cavaliere 100, 00133 Rome, Italy \\ 5 Centro di Ateneo di Studi ed Attività Spaziali "Giuseppe Colombo" (CISAS), Università di Padova, Via Venezia 15, 35131 \\ Padova, Italy \\ ${ }^{6}$ IFAC - CNR, Via Madonna del Piano 10, 50019 Sesto Fiorentino, Firenze, Italy \\ ${ }^{7}$ ESA NEO Coordination Centre, Largo Galileo Galilei, 1, 00044 Frascati (RM), Italy \\ 8 Agenzia Spaziale Italiana, Via del Politecnico 1, 00133 Rome, Italy
}

Received 18 July 2020 / Accepted 16 October 2020

\begin{abstract}
Context. The near-Earth objects (NEOs), whose proximity makes them the most accessible bodies in the Solar System, allow us to sample asteroids from tens of kilometers down to objects of a few meters in size. However, while the physical properties for the largest bodies are mostly known, we have very little physical information regarding the small NEOs. These objects today represent the overall majority among the $\sim 2500$ new discoveries each year, but they are usually only bright enough to be observable during their close approaches.

Aims. Our aim was to extend our survey that started in 2015 on the NEO population, using ground-based observations to characterize the fainter (and typically smaller) NEOs observable each night.

Methods. We performed BVRIz photometry of NEOs, making use of the DOLORES instrument at the Telescopio Nazionale Galileo (TNG, La Palma, Spain) and the Asiago Schmidt telescope (Italy), in order to derive visible color indexes and the taxonomic classification for each target in our sample.

Results. We taxonomically classified 51 new NEOs for the first time. Together with data obtained in our previous work and collected by other surveys available online, we analyzed an extended sample of 1081 individual NEOs. While the overall majority of them belong to the S-complex, our analysis of the taxonomic distribution found a larger contribution for dark bodies going toward larger $\mathrm{H}$, suggesting that they could be more abundant among the fainter NEOs. Moreover, we find an interesting correlation between semi-major axis and diameter, which could be in part related to the Yarkovsky effect. Rapid characterization of the fainter NEO population shortly after their discovery will be crucial in the future, before those bodies become too faint to be observed, or lost forever.
\end{abstract}

Key words. minor planets, asteroids: individual: NEOs - techniques: photometric - surveys

\section{Introduction}

The study of near-Earth objects (NEOs) has opened new frontiers in planetary sciences in the last decades due to the fact that their proximity makes them the most accessible bodies in our Solar System. Their investigation can offer answers to several pressing questions in modern planetology (regarding, e.g., planetary formation, delivery of water and organics to the early Earth, and emergence of life). Moreover, water and valued minerals stored inside NEOs have already intrigued governments and private companies, allured by the idea of obtaining resources through asteroid mining (Calla et al. 2018). Futhermore, NEOs represent a risk for future human civilization, since some of them can be potential impactors (Perna et al. 2013a). For all these reasons, it is clear that the characterization of NEO physical properties is essential from both ground and space.

\footnotetext{
* Table A.1 is only available at the CDS via anonymous ftp to cdsarc.u-strasbg.fr (130.79.128.5) or via http://cdsarc. u-strasbg.fr/viz-bin/cat/J/A+A/644/A23
}

Space missions have already visited and characterized a handful of NEOs: NASA NEAR Shoemaker (Veverka et al. 2000) and the JAXA Hayabusa mission (Saito et al. 2006) have orbited two silicate bodies, (433) Eros and (25143) Itokawa, respectively. Two missions recently analyzed a primitive NEO in detail: the JAXA space mission Hayabusa2 visited the asteroid (162173) Ryugu (Watanabe et al. 2017), while NASA OSIRISREx mission is at the moment attempting to retrieve a sample from the surface of (101955) Bennu (Lauretta et al. 2017). In the fall of 2022, the NASA DART mission (Cheng et al. 2018) will attempt the first demonstration of a kinetic impact on the Didymos binary system. The ASI LICIACube cubesat (Dotto et al. 2020), hosted as a piggyback, will be released ten days before the impact and will characterize the crater, the plume, and the non-impacted size. However, a limited number of targets can be characterized from space. For all NEOs that will never be a target of a mission, ground-based observations are crucial to deriving physical information. Moreover, a large database of physical properties, achieved mainly from ground observations, is the only way to derive a statistically significant sample. 
As of October 2020, 24000 NEOs have been discovered. However, we know the physical properties for a very limited amount, with the situation being unbalanced in favor of larger bodies: roughly a $30 \%$ of the objects with a diameter $>1 \mathrm{~km}$, and only a few percent of those with diameters below a few hundred meters have some kind of physical characterization (albedo, color indexes, spectra, radar, etc.). The situation is getting progressively worse since the current discovery rate of NEOs is about 2500 new objects each year, and the overall majority of these new discoveries regards the "small" NEO population. These are usually bright enough to be observable only during their close approach, making it clear that a rapid physical characterization is crucial, before they become too faint or they are lost forever.

The physical characterization of NEOs is also important due to the great diversity in their composition: the overall majority of NEOs belongs to the S-complex, having experienced a certain degree of thermal evolution, while according to literature only $15 \%$ of bodies resemble a more pristine carbonaceous composition (Binzel et al. 2015). It is known that an observational bias can be at play, due to the fact that both discovery and spectrophotometric surveys are magnitude limited, hence more sensitive to bright objects (like S-type bodies) than dark, low-albedo ones (like C-type objects). Thermal infrared surveys, which are less sensitive to the observational bias, estimated for the low-albedo asteroids a total contribution to the NEO population of approximately 30\% (Mainzer et al. 2015). This suggests a possible underestimation of carbonaceous bodies among the NEOs.

In order to better understand the actual taxonomic distribution of NEOs, we started a photometric survey to characterize the NEO population. During the first year of observations (Ieva et al. 2018), we found a possible lack of carbonaceous material going to smaller sizes. While this could be related to the aforementioned observational bias, several authors have suggested that a physical mechanism could have a non-negligible effect: Delbo et al. (2014) invoked the higher efficiency of thermal fragmentation for darker bodies, which could ultimately result in a faster destruction; Scheeres (2018) hypothesized that the porous carbonaceous bodies have higher mechanical resistance to repeated rotational fissions.

We focused our second year of observations on the characterization of the faintest NEOs observable each night. Here we present new photometric color indexes and a taxonomic classification for 51 objects observed by our group during a longterm survey at the Telescopio Nazionale Galileo (TNG) and at the Asiago Schmidt telescope, within the framework of the NEOShield-2 project ${ }^{1}$. In the following sections, we describe the observational setup and the reduction procedures (Sect. 2), we present our derived taxonomy (Sect. 3), and we give our analysis (Sect. 4) using a much larger sample. We also identify potential future targets for space missions. Finally, we discuss our results in a broader context (Sect. 5).

\section{Observations and data reduction}

The data presented in this work were collected at the TNG (La Palma, Canary Islands, Spain) and at the Asiago 67/92 cm Schmidt telescope (Italy) between April 2016 and September 2017. Targets were chosen among the faintest ones observable each night, to extend our physical characterization toward fainter (and typically smaller) NEOs. Observational conditions are reported in Table 1.

\footnotetext{
1 https://www.neoshield.eu
}

Table 1. Observational circumstances for the sample of 51 observed NEOs.

\begin{tabular}{|c|c|c|c|c|c|}
\hline Object & Date & Telescope & $\begin{array}{c}\Delta \\
(\mathrm{au})\end{array}$ & $\begin{array}{c}r \\
(\mathrm{au})\end{array}$ & $\alpha$ \\
\hline (7350) $1993 \mathrm{VA}$ & $25 / 04 / 2016$ & Asiago & 0.304 & 1.197 & 45.2 \\
\hline (23187) 2000 PN9 & $27 / 04 / 2016$ & Asiago & 0.849 & 1.339 & 48.7 \\
\hline (68950) 2002 QF15 & 29/10/2016 & Asiago & 0.236 & 1.017 & 77.5 \\
\hline (137032) 1998 UO1 & 29/10/2016 & Asiago & 0.481 & 1.146 & 59.5 \\
\hline (137170) $1999 \mathrm{HF} 1$ & $27 / 04 / 2016$ & Asiago & 0.577 & 1.002 & 73.8 \\
\hline (138847) 2000 VE62 & $29 / 04 / 2016$ & Asiago & 0.347 & 1.158 & 56.1 \\
\hline (163243) 2002 FB3 & 29/04/2016 & Asiago & 0.775 & 1.204 & 56.3 \\
\hline (164207) 2004 GU9 & $27 / 02 / 2017$ & TNG & 0.277 & 1.131 & 53.3 \\
\hline (242191) 2003 NZ6 & $27 / 05 / 2017$ & TNG & 0.374 & 1.116 & 64.6 \\
\hline (265482) $2005 \mathrm{EE}$ & $27 / 02 / 2017$ & TNG & 0.241 & 1.205 & 24.2 \\
\hline (265962) $2006 \mathrm{CG}$ & $24 / 07 / 2017$ & TNG & 0.334 & 1.309 & 24.8 \\
\hline (304640) 2006 WW1 & $24 / 07 / 2017$ & TNG & 0.219 & 1.057 & 73.3 \\
\hline (317255) 2002 DJ5 & $27 / 02 / 2017$ & TNG & 0.402 & 1.392 & 2.8 \\
\hline (326683) $2002 \mathrm{WP}$ & $17 / 01 / 2017$ & Asiago & 0.312 & 1.173 & 46.4 \\
\hline (413002) 1999 VG22 & $27 / 05 / 2017$ & TNG & 0.632 & 1.496 & 31.6 \\
\hline (414960) 2011 CS4 & 30/09/2016 & TNG & 0.215 & 1.095 & 58.9 \\
\hline (420738) 2012 TS & $30 / 09 / 2016$ & TNG & 0.222 & 1.100 & 58.4 \\
\hline (467460) 2006 JF42 & $27 / 05 / 2017$ & TNG & 0.285 & 1.049 & 75.1 \\
\hline (467963) 2012 JT17 & $10 / 11 / 2016$ & Asiago & 0.216 & 1.199 & 13.5 \\
\hline (474425) 2002 YF4 & $27 / 05 / 2017$ & TNG & 0.521 & 1.455 & 26.1 \\
\hline (479325) 2013 TV5 & $30 / 10 / 2016$ & Asiago & 0.247 & 1.023 & 76.2 \\
\hline (481394) 2006 SF6 & $24 / 07 / 2017$ & TNG & 0.168 & 1.019 & 84.0 \\
\hline (483856) 2008 ER7 & $27 / 02 / 2017$ & TNG & 0.286 & 1.272 & 8.7 \\
\hline (486739) 2014 EW24 & 20/01/2017 & Asiago & 0.198 & 1.113 & 45.3 \\
\hline (488803) 2005 GB120 & 01/05/2017 & TNG & 0.181 & 1.099 & 55.7 \\
\hline (489337) 2006 UM & $29 / 10 / 2016$ & Asiago & 0.389 & 1.364 & 15.0 \\
\hline (494710) 2005 MO13 & $27 / 05 / 2017$ & TNG & 0.236 & 1.218 & 27.0 \\
\hline (495222) 2013 GU38 & $27 / 05 / 2017$ & TNG & 0.346 & 1.202 & 49.7 \\
\hline (497025) 2003 QW30 & $24 / 07 / 2017$ & TNG & 0.408 & 1.345 & 30.7 \\
\hline (499490) $2010 \mathrm{MW}$ & $24 / 07 / 2017$ & TNG & 0.495 & 1.354 & 38.7 \\
\hline (503293) 2016 AA9 & $24 / 07 / 2017$ & TNG & 0.599 & 1.481 & 31.1 \\
\hline (522684) $2016 \mathrm{JP}$ & $27 / 05 / 2017$ & TNG & 0.293 & 1.187 & 47.7 \\
\hline $1999 \mathrm{VT}$ & $29 / 10 / 2016$ & Asiago & 0.228 & 1.207 & 18.3 \\
\hline 2003 EZ16 & $27 / 02 / 2017$ & TNG & 0.205 & 1.191 & 11.6 \\
\hline 2005 CL & $22 / 10 / 2016$ & TNG & 0.592 & 1.567 & 12.0 \\
\hline $2005 \mathrm{TF}$ & $30 / 09 / 2016$ & TNG & 0.266 & 1.256 & 14.6 \\
\hline 2006 KA40 & $24 / 07 / 2017$ & TNG & 0.348 & 1.219 & 47.2 \\
\hline 2013 CL118 & $27 / 02 / 2017$ & TNG & 0.357 & 1.311 & 22.7 \\
\hline 2013 FY13 & $22 / 10 / 2016$ & TNG & 0.457 & 1.431 & 14.2 \\
\hline 2014 DH23 & $27 / 02 / 2017$ & TNG & 0.302 & 1.289 & 8.2 \\
\hline 2015 YR1 & $24 / 07 / 2017$ & TNG & 0.523 & 1.323 & 44.2 \\
\hline 2016 GD135 & $30 / 09 / 2016$ & TNG & 0.225 & 1.187 & 31.4 \\
\hline 2016 UU80 & 27/02/2017 & TNG & 0.199 & 1.178 & 17.9 \\
\hline 2016 YU8 & $27 / 05 / 2017$ & TNG & 0.306 & 1.202 & 46.1 \\
\hline 2017 AC5 & 01/05/2017 & TNG & 0.269 & 1.212 & 36.1 \\
\hline 2017 CS & $27 / 05 / 2017$ & TNG & 0.022 & 1.019 & 74.7 \\
\hline 2017 HN3 & $27 / 05 / 2017$ & TNG & 0.175 & 1.127 & 46.0 \\
\hline 2017 HY50 & $27 / 05 / 2017$ & TNG & 0.290 & 1.247 & 32.1 \\
\hline 2017 JF3 & $27 / 05 / 2017$ & TNG & 0.155 & 1.059 & 68.8 \\
\hline 2017 MY2 & $24 / 07 / 2017$ & TNG & 0.252 & 1.250 & 19.2 \\
\hline 2017 OP68 & 21/09/2017 & TNG & 0.094 & 1.089 & 23.7 \\
\hline
\end{tabular}

Notes. $\Delta$ and $r$ are the topocentric and heliocentric distances, respectively. $\alpha$ is the solar phase angle.

At TNG, observations were carried out using the Device Optimized for the LOw RESolution (DOLORES), equipped with a $2048 \times 2048$ E2V 4240 thinned, back-illuminated, deepdepleted, Astro-BB coated CCD with a pixel size of $13.5 \mu \mathrm{m}$. 
At Asiago, observations were performed using the $67 / 92 \mathrm{~cm}$ Schmidt telescope equipped with an ON-Semi KAF-16803 $\mathrm{CCD}$, with an active area of $4096 \times 4096$ pixels, a scale of 0.87 arcsec/pixel and a FOV of $59 \times 59$ arcmin. We used the broadband $B-V-R-I$ filters, and occasionally the $z$ filter. We adjusted the exposure time to obtain a $\mathrm{S} / \mathrm{N}$ of at least 30 in $B V R I$ filters, and of at least 20 in the $z$ filter, in order to reach a high level of precision in the magnitude determination able to differentiate taxonomy in visible wavelengths.

Images were reduced with the MIDAS software package following the same approach described in Ieva et al. (2018). This includes bias subtraction, flat-field correction, measurement of instrumental magnitude via aperture photometry, removal of the sky contribution, and absolute calibration via the observation of standard stars. Magnitudes for TNG data were independently confirmed using the photometry pipeline (PP; Mommert 2017).

We reduced photometric measurements for a total sample of 51 individual NEOs. A few of them have also been observed by the MITHNEOS MIT-Hawaii Near-Earth Object Spectroscopic Survey (Binzel et al. 2019). However, MITHNEOS observations were performed in the near-infrared range $(0.8-2.4 \mu \mathrm{m})$, thus we extended the wavelength coverage in the visible for those present in both surveys.

\section{Taxonomic classification}

In order to derive a taxonomic classification for all the objects considered in our sample, first we obtained the $B-V, V-R$, $V-I$ and $V-z$ colors from $B, V, R, I$, and $z$ magnitudes. Then, we converted our color indexes into reflectance values (normalized at unity at the $V$ filter), and we rescaled the $B, R, I$, and $z$ reflectance using:

$\mathcal{R}_{\lambda}=10^{-0.4\left[\left(M_{\lambda}-V\right)-\left(M_{\lambda}-V\right)_{\odot}\right]}$

where $\left(M_{\lambda}-V\right)$ and $\left(M_{\lambda}-V\right)_{\odot}$ are the colors for the object and the Sun at the wavelength $\lambda$, respectively. We compared them with sample spectra taken from the Bus-DeMeo taxonomy (DeMeo et al. 2009) using standard curve-matching techniques to establish the best-fit spectral class.

For our taxonomy, we only considered major groupings: the S-complex (including S-, Sa-, Sq-, Sr-, Sv-, and Q-type objects); the C-complex (B-, C-, Cb-, Cg-, Cgh-, and Ch-type objects); the X-complex (X-, Xc-, Xe-, Xk-, and Xn-type objects). Classes among complexes are typically distinguished using subtle features present in the Bus-DeMeo classification, which requires high-resolution spectra. Moreover, this will encompass more objects per group, allowing a more comprehensive statistic. Few NEOs were also classified as other end-members (A- and V-type objects). The final obtained colors and derived taxonomic classification are shown in Table 2: 29 bodies were classified as S-complex, 13 as C-complex, and six as X-complex objects; the remaining belong to the A- or V-type.

Using the same approach as in our previous work, we derived average $B-R$ and $V-I$ colors for taxonomic groups considered in our sample, and we compared these values with the same colors obtained from the template spectra of the Bus-DeMeo taxonomy (see Table 3). For C-, S-, and X-complex objects, we found a general agreement, with these classes having average colors similar to the ones derived from template spectra. The greater dispersion shown for $\mathrm{V}$-type bodies, particularly for $V-I$ color, could be related to the limited available sample (only two objects) or

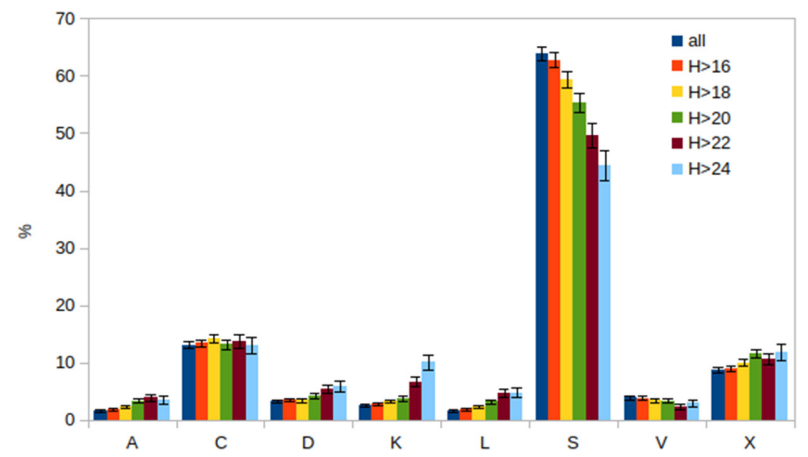

Fig. 1. Percentage distribution of taxonomic complexes and classes in our survey, together with data collected from Perna et al. (2018), Devogèle et al. (2019), and the MITHNEOS campaign, shown according to a limiting $\mathrm{H}$. Error bars were computed using a standard Poisson approach.

with the intrinsic greater spectral variation of the V-type class (see also Ieva et al. 2016).

\section{Data analysis}

\subsection{Dependence on the absolute magnitude $H$}

The absolute magnitude $\mathrm{H}$ can be a useful proxy to compare the intrinsic brightness of an NEO, and, assuming reasonable constraints on the albedo, a possible tool to estimate their size, which is a key quality for many scientific purposes (e.g., designing a successful mitigation scenario). The albedo is, however, available only for a limited sample. Therefore, it is useful to begin our analysis by comparing the effects of $\mathrm{H}$ for the different taxonomic classes and complexes considered in this work.

The whole sample of NEOs observed by our group between 2014 and 2017 (Ieva et al. 2018 and this work) consists of 118 new NEOs. In order to enlarge the statistics and strengthen our results, we considered for this analysis, together with our sample, objects characterized in Perna et al. (2018), in Devogèle et al. (2019), and all the NEOs observed by the MITHNEOS campaign and available on their website ${ }^{2}$. In order to be consistent, we taxonomically reclassified all the NEO spectra taken from other surveys using the same curve-matching technique approach adopted for our original sample. Sometimes, comparing visible (VIS) and near-infrared (NIR) data can lead to a disproportion in the taxonomic classes, due to the fact that the same object can have slightly different taxonomies based on VIS or NIR spectra only. However, almost $15 \%$ of our sample (160 NEOs) has been observed both in the VIS and NIR, leading to a similar taxonomic classification. Moreover, the remaining NIR-only and VIS-only are detected almost in the same proportions, accounting, respectively, for 531 and 390 NEOs, therefore reassuring us that the relative class abundances are not being distorted.

Final taxonomic classes are reported in Table A.1. The final sample consists of 1081 individual NEOs: 64\% (692 NEOs) belong to the S-complex, 13\% and 9\% (142 and 95 bodies, respectively) are classified as $\mathrm{C}$ - and $\mathrm{X}$-complex types; some objects were found belonging to the V- (42), D- (36), K- (29), L- (18), and A-type (17); finally, ten bodies were recognized as belonging to one of the rare classes (O-, R- and T-type). In Fig. 1, we report the distribution of the taxonomic classes considered here according to the limiting absolute magnitude $\mathrm{H}$. It is possible to see that a trend with the increasing $\mathrm{H}$ appears for the

2 http://smass.mit.edu/minus.html 
Table 2. Visual $(V)$ magnitude, $B-V, V-R, V-I$, and $V-z$ color indexes and the obtained taxonomy for our original sample of 51 NEOs.

\begin{tabular}{|c|c|c|c|c|c|c|}
\hline Object & $V$ & $B-V$ & $V-R$ & $V-I$ & $V-z$ & Taxonomy \\
\hline$(7350)$ & $16.69 \pm 0.10$ & $0.67 \pm 0.20$ & $0.35 \pm 0.20$ & $0.62 \pm 0.12$ & & C-complex \\
\hline$(23187)^{*}$ & $8.11 \pm 0.10$ & $0.83 \pm 0.20$ & $0.49 \pm 0.20$ & & & S-complex \\
\hline$(68950)^{*}$ & $6.01 \pm 0.10$ & $0.74 \pm 0.21$ & $0.44 \pm 0.17$ & $0.97 \pm 0.21$ & & S-comples \\
\hline (137032) & $7.31 \pm 0.09$ & $0.97 \pm 0.16$ & $0.25 \pm 0.21$ & $0.65 \pm 0.15$ & & S-comples \\
\hline (137170) & $16.13 \pm 0.10$ & $0.65 \pm 0.08$ & $0.37 \pm 0.07$ & $0.86 \pm 0.10$ & & $\mathrm{X}$-comple \\
\hline 38847) & $17.15 \pm 0.10$ & $1.00 \pm 0.22$ & $0.42 \pm 0.20$ & $0.70 \pm 0.20$ & $\ldots$ & S-comples \\
\hline$(163243)^{*}$ & $18.15 \pm 0.10$ & $0.86 \pm 0.20$ & $0.63 \pm 0.20$ & $0.94 \pm 0.20$ & & S-comple \\
\hline$(164207)^{*}$ & $20.45 \pm 0.04$ & $0.87 \pm 0.10$ & & $0.71 \pm 0.12$ & & S-complex \\
\hline (242191) & $.74 \pm 0.04$ & $0.92 \pm 0.11$ & $0.31 \pm 0.08$ & $0.72 \pm 0.10$ & $0.44 \pm 0.10$ & S-complex \\
\hline$(265482)^{*}$ & $.68 \pm 0.03$ & $0.78 \pm 0.08$ & $0.60 \pm 0.06$ & $0.51 \pm 0.10$ & $\ldots$ & S-comple \\
\hline (265962) & $8.37 \pm 0.04$ & $0.83 \pm 0.11$ & $0.27 \pm 0.10$ & $0.80 \pm 0.09$ & $0.43 \pm 0.09$ & $\mathrm{X}$-comple \\
\hline (304640) & $.75 \pm 0.06$ & $0.88 \pm 0.13$ & $0.59 \pm 0.11$ & $0.99 \pm 0.11$ & $0.58 \pm 0.12$ & A-type \\
\hline 317255$)^{*}$ & $18.83 \pm 0.04$ & $0.75 \pm 0.10$ & $0.37 \pm 0.08$ & $0.62 \pm 0.10$ & & C-comples \\
\hline & $17.76 \pm 0.07$ & $0.93 \pm 0.17$ & $0.47 \pm 0.14$ & $0.81 \pm 0.15$ & $\ldots$ & S-complex \\
\hline 13002$)$ & $20.27 \pm 0.05$ & $1.02 \pm 0.13$ & $0.55 \pm 0.09$ & $0.77 \pm 0.13$ & $\cdots$ & S-complex \\
\hline 14960) & $8.57 \pm 0.05$ & $0.71 \pm 0.10$ & $0.40 \pm 0.12$ & $0.74 \pm 0.07$ & & $\mathrm{X}$-comple \\
\hline (420738) & $20.00 \pm 0.07$ & $0.68 \pm 0.13$ & $0.39 \pm 0.14$ & & & $\mathrm{X}$-comple \\
\hline 160)* & .06 & $0.78=$ & $0.46 \pm 0$ & $0.50 \pm 0.14$ & $0.24 \pm 0.13$ & S-complex \\
\hline 7963) & $16.58 \pm 0.10$ & & $0.55 \pm 0.20$ & $0.90 \pm 0.20$ & ( & S-complex \\
\hline$(474425)$ & $19.41 \pm 0.04$ & $0.92 \pm 0.09$ & $0.38 \pm 0.08$ & $0.71 \pm 0.10$ & $0.18 \pm 0.09$ & S-complex \\
\hline (479325) & $8.33 \pm 0.07$ & $1.03 \pm 0.14$ & $0.50 \pm 0.18$ & $0.70 \pm 0.32$ & $\ldots$ & S-complex \\
\hline (481394 & $19.19 \pm 0.05$ & $1.12 \pm 0.12$ & $0.41 \pm 0.09$ & $0.66 \pm 0.12$ & $\ldots$ & S-complex \\
\hline & $20.62 \pm 0.04$ & $0.79 \pm$ & $0.43 \pm 0.08$ & $0.55 \pm 0.12$ & $\ldots$ & \\
\hline 186739) & $17.94 \pm 0.06$ & $0.84 \pm 0.13$ & $0.31 \pm 0.12$ & $0.63 \pm 0.12$ & & C-complex \\
\hline (488803) & $19.07 \pm 0.07$ & $0.95 \pm 0.25$ & $0.63 \pm 0.11$ & $0.85 \pm 0.14$ & $0.38 \pm 0.14$ & S-complex \\
\hline (489337) & $17.65 \pm 0.10$ & $0.69 \pm 0.20$ & $0.49 \pm 0.20$ & $0.77 \pm 0.20$ & & S-con \\
\hline & $19.28 \pm$ & & $0.40 \pm 0$ & $0.67 \pm 0.10$ & $0.40 \pm 0.09$ & C-col \\
\hline (495222) & $20.61 \pm 0.07$ & $0.77 \pm 0$ & $0.55 \pm 0.14$ & $0.83 \pm 0.17$ & & S-con \\
\hline (497025) & $20.74 \pm 0.06$ & $0.91 \pm 0.15$ & $0.53 \pm 0.11$ & $0.69 \pm 0.14$ & $0.54 \pm 0.14$ & S-con \\
\hline (499490) & $9.95 \pm 0.05$ & $0.82 \pm 0.11$ & $0.30 \pm 0.11$ & $0.61 \pm 0.12$ & $0.23 \pm 0.12$ & $\mathrm{C}-\mathrm{co}$ \\
\hline & $0.54 \pm 0.07$ & & $0.55 \pm 0.14$ & $0.96 \pm 0.13$ & $0.61 \pm 0.14$ & S-col \\
\hline$(522684)^{*}$ & $20.58 \pm 0.04$ & $0.90 \pm$ & $5 \pm 0.09$ & $0.68 \pm 0.11$ & $0.01 \pm 0.21$ & V-type \\
\hline 1999 VT & $17.82 \pm 0.08$ & $0.75 \pm 0.14$ & $0.36 \pm 0.14$ & $0.66 \pm 0.04$ & $\ldots$ & C-complex \\
\hline 2003 EZ16 & $20.40 \pm 0.04$ & $0.63 \pm 0.12$ & $0.44 \pm 0.08$ & $0.63 \pm 0.10$ & & S-col \\
\hline $2005 \mathrm{CL}^{*}$ & $0.93 \pm 0.05$ & & $0.49 \pm 0.10$ & $0.67 \pm 0.13$ & $\cdots$ & S-co \\
\hline $2005 \mathrm{TF}$ & $.62 \pm 0.09$ & $0.78 \pm 0.13$ & $0.41 \pm 0.14$ & $0.66 \pm 0.14$ & & S-complex \\
\hline 2006 KA40 & $19.22 \pm 0.05$ & $0.95 \pm 0.11$ & $0.44 \pm 0.10$ & $0.45 \pm 0.13$ & $0.10 \pm 0.10$ & V-type \\
\hline 2013 CL118 & $20.45 \pm 0.03$ & $0.64 \pm 0.08$ & $0.38 \pm 0.07$ & $0.69 \pm 0.10$ & $0.32 \pm 0.35$ & C-complex \\
\hline 2013 FY13 & $20.37 \pm 0.07$ & & $0.42 \pm 0.17$ & $0.96 \pm 0.19$ & & X-complex \\
\hline 2014 DH23 & $20.04 \pm 0.06$ & $0.83 \pm 0.13$ & $0.43 \pm 0.12$ & $0.47 \pm 0.23$ & $\cdots$ & S-complex \\
\hline 2015 YR1 & $19.86 \pm 0.06$ & $0.63 \pm 0.13$ & $0.33 \pm 0.12$ & $\ldots$ & $\ldots$ & C-complex \\
\hline 2016 GD135* & $20.34 \pm 0.08$ & $\ldots$ & $0.60 \pm 0.15$ & $0.71 \pm 0.14$ & & S-complex \\
\hline 2016 UU80 & $18.76 \pm 0.03$ & $0.96 \pm 0.07$ & $0.52 \pm 0.07$ & $0.96 \pm 0.08$ & & S-complex \\
\hline 2016 YU8 & $19.71 \pm 0.03$ & $0.71 \pm 0.08$ & $0.41 \pm 0.07$ & $0.78 \pm 0.09$ & $1.11 \pm 0.08$ & $\mathrm{X}$-complex \\
\hline 2017 AC5 & $18.86 \pm 0.03$ & $0.76 \pm 0.07$ & $0.46 \pm 0.06$ & $0.81 \pm 0.08$ & $0.29 \pm 0.09$ & S-complex \\
\hline 2017 CS* & $18.55 \pm 0.04$ & $0.75 \pm 0.09$ & $0.31 \pm 0.08$ & $0.76 \pm 0.09$ & $\ldots$ & C-complex \\
\hline 2017 HN3 & $19.79 \pm 0.04$ & $0.92 \pm 0.11$ & $0.35 \pm 0.08$ & $0.68 \pm 0.11$ & $0.43 \pm 0.10$ & C-complex \\
\hline 2017 HY50* & $19.10 \pm 0.04$ & $0.66 \pm 0.09$ & $0.33 \pm 0.08$ & $0.70 \pm 0.09$ & $0.42 \pm 0.09$ & C-complex \\
\hline 2017 JF3* & $20.20 \pm 0.04$ & $0.89 \pm 0.11$ & $0.55 \pm 0.08$ & $0.80 \pm 0.12$ & & S-complex \\
\hline 2017 MY2 & $19.50 \pm 0.04$ & $0.60 \pm 0.09$ & $0.31 \pm 0.08$ & $0.67 \pm 0.09$ & $0.23 \pm 0.09$ & C-complex \\
\hline 2017 OP68 & $17.00 \pm 0.03$ & $0.78 \pm 0.07$ & $0.38 \pm 0.06$ & $0.64 \pm 0.09$ & $0.28 \pm 0.07$ & C-complex \\
\hline
\end{tabular}

Notes. Objects with an asterisk are PHAs, as defined in the text.

low-albedo population, with the rare D-type bodies almost doubling from $3 \%$ when considering the whole sample, to $6 \%$ for $H>24$. At the same time, the K- and L-type relative contribution increases when sampling fainter objects, going from a mere $4 \%$ to almost $15 \%$. Even the contribution for the X-complex seems to increase for larger $\mathrm{H}$, from 9 to $12 \%$. Carbonaceous C-complex bodies, however, seem to steadily remain around $13-14 \%$. On the contrary, we noticed a decrease in silicate S-complex bodies, passing from $64 \%$ in the overall survey to $44 \%$ for $H>24$, hence confirming the latest findings of 
Table 3. Average $B-R$ and $V-I$ color ranges obtained in our sample and using the Bus-DeMeo sample spectra.

\begin{tabular}{lcccc}
\hline \hline & $(B-R)_{\mathrm{ave}}$ & $(B-R)_{\mathrm{BDM}}$ & $(V-I)_{\mathrm{ave}}$ & $(V-I)_{\mathrm{BDM}}$ \\
\hline $\mathrm{C}$ & $1.08 \pm 0.10$ & $1.09_{-0.09}^{+0.11}$ & $0.65 \pm 0.03$ & $0.69_{-0.10}^{+0.04}$ \\
$\mathrm{X}$ & $1.09 \pm 0.05$ & $1.13_{-0.07}^{+0.12}$ & $0.82 \pm 0.07$ & $0.78_{-0.04}^{+0.06}$ \\
$\mathrm{~S}$ & $1.35 \pm 0.13$ & $1.29_{-0.11}^{+0.14}$ & $0.74 \pm 0.14$ & $0.82_{-0.14}^{+0.10}$ \\
$\mathrm{~V}$ & $1.37 \pm 0.02$ & $1.34_{-0.09}^{+0.09}$ & $0.57 \pm 0.12$ & $0.75_{-0.08}^{+0.08}$ \\
\hline
\end{tabular}

Notes. Errors for our sample take into account the standard deviation for each taxonomic group, while for Bus-DeMeo spectra, we considered the dispersion of each sample spectrum.

Table 4. Taxonomic distribution in our enlarged survey of NEOs.

\begin{tabular}{lcccccc}
\hline \hline Tax. class & All & $H>16$ & $H>18$ & $H>20$ & $H>22$ & $H>24$ \\
\hline A & 17 & 17 & 17 & 16 & 9 & 6 \\
C & 142 & 132 & 108 & 66 & 35 & 22 \\
D & 36 & 34 & 26 & 21 & 14 & 10 \\
K & 29 & 28 & 25 & 19 & 17 & 17 \\
L & 18 & 18 & 18 & 16 & 12 & 8 \\
S & 692 & 616 & 451 & 278 & 127 & 75 \\
V & 42 & 38 & 26 & 17 & 6 & 5 \\
X & 95 & 87 & 76 & 58 & 27 & 20 \\
\hline Total & 1081 & 980 & 757 & 501 & 254 & 169 \\
\hline
\end{tabular}

Devogèle et al. (2019) using a much broader sample. Interestingly, all of the A-types considered here are found for $H>19$, suggesting that those elusive olivine-dominated assemblages can be extremely faint (see details in Table 4). This is in agreement with the "battered-to-bits" scenario proposed by Burbine et al. (1996) to explain the apparent lack of mantle material of differentiated parent bodies within the current asteroid population.

In our extended survey, roughly $15 \%$ features objects with a $H>24$. This is mostly because for these NEOs the discovery apparition provides the best observing conditions for many years. If they are not characterized by then, their next observable window could even be decades apart, or worse: they are lost forever. Despite the limited amount of faint NEOs in our survey, our analysis clearly shows a trend of increasing darker (mainly D-, K- and L-type) and decreasing brighter (mainly S-complex) NEOs going toward higher $\mathrm{H}$.

\subsection{Dependence on the size}

In order to obtain a reliable estimation of the target size, we used the combination of the absolute magnitude $\mathrm{H}$ and the geometrical albedo $\rho$. Since albedo is only available for roughly $1 / 4$ of the sample (265 NEOs), for each class or complex we derived an average albedo among the tabulated ones and we used it for the whole taxonomic group. As a further control, we also checked that average albedos are in agreement with estimated values for each class reported in Ryan \& Woodward (2010). The only discrepancy is for the A-class, presumably due to the fact that only one object has a computed albedo. Final diameters and albedos for objects characterized in our enlarged sample are reported in Table A.1.

To perform a study of the possible correlations between size and taxa in our sample, we sorted it into size bins with comparable amounts of objects in each group. An ideal selection, aiming to increase the statistical significance of our results, will be to consider a large number of NEOs in each bin. We selected them in order to have roughly 180 objects in each bin (i.e., approximately $1 / 6$ of the sample). Simulations were repeated many times using several different thresholds, showing that final results did not change appreciably, thus boosting our confidence in our original choice. NEOs in our enlarged sample were therefore sorted according to their estimated size in six dimensional bins: $D<60 \mathrm{~m}$ (181), $60 \mathrm{~m}<D<200 \mathrm{~m}$ (180), $200 \mathrm{~m}<D<400$ m (176), $400 \mathrm{~m}<D<700 \mathrm{~m}$ (180), $700 \mathrm{~m}<D<1300 \mathrm{~m}$ (184), and $D>1300 \mathrm{~m}(180)$.

Analyzing the three main represented groups (C-, S- and $\mathrm{X}$-complex), it is clear that not all size bins contribute in the same way (Table 5): compared to the overall representation, carbonaceous NEOs seem to be slightly underrepresented below $D<400 \mathrm{~m}$, particularly in the $200-400 \mathrm{~m}$ bin, where they contribute only by a mere $6.8 \%$. This could be in part related to the fact that their lower average albedo (with respect, e.g., to silicate bodies) will eventually result in a larger estimated dimension. At the same time, silicate $\mathrm{S}$-complexes essentially remain stable between $60<D<1300 \mathrm{~m}$, with a contribution between 65.9 and $71.1 \%$, which is very similar to its overall $63.9 \%$ contribution. In the smallest range, the dimensional analysis is strongly influenced by the noticeable lack of fainter $(H>24)$ S-type bodies (see Sect. 4.1), while in the largest range $(D>1300 \mathrm{~m})$ the higher average albedo of silicates favors the presence of carbonaceous bodies in this bin, resulting in an increase of the latter and a deficiency of S-complex NEOs.

The X-complex contribution to the NEO population is close to the one estimated from the whole sample $(\sim 9 \%)$; interestingly, we saw a higher contribution among the $200-400 \mathrm{~m}$ bin and a lower contribution in the 700-1300 m bin. While further data could help improve the X-complex representation, it is worth mentioning here that the X-class in the DeMeo taxonomy comprehends both low- and high-albedo bodies. The use of an average albedo in this particular case should be taken with precaution.

\subsection{Dependence on the orbital parameters}

We looked for possible relationships between taxonomical classes and orbital parameters. Median values for the latter (semi-major axis $a$, eccentricity $e$, inclination $i$, perihelion $q$, aphelion $Q$, and minimum orbit intersection distance (MOID) with our planet) are reported in Table 6 . Using a much broader sample than the one reported in Ieva et al. (2018), we confirmed that silicate S-complex bodies show a lower median value for $a$ and $q$ when compared to darker and more primitive NEOs (C- and D-types). This might be related to the scenario of silicate bodies formed mostly in the inner main belt, closer to powerful resonances able to pump them into near-Earth orbits, as recently shown by dynamical analysis on the NEO population (Binzel et al. 2019). Similarly, low-albedo C- and D-type NEOs show hints of originating from the middle and outer main belt (Granvik \& Brown 2018), with those primitive bodies having the highest $a$ and $Q$ of the whole sample. Interestingly, all the A-type NEOs classified in this work show a low inclination below $9.5^{\circ}$. Due to the limited amount of A-type bodies available in our sample (and in literature; see Sanchez et al. 2014), further data are desired to confirm this peculiar result.

We also investigated the relation between taxa and perihelium. According to some recent theoretical models, there are fewer NEOs at smaller perihelium distances than the ones 
Table 5. Taxonomic distribution according to estimated diameter for the C-, S-, and X-complex.

\begin{tabular}{lc|cccccc}
\hline \hline Tax. class & $\begin{array}{c}\text { All } \\
(1081)\end{array}$ & $\begin{array}{c}D<60 \mathrm{~m} \\
(181)\end{array}$ & $\begin{array}{c}D 60-200 \mathrm{~m} \\
(180)\end{array}$ & $\begin{array}{c}D 200-400 \mathrm{~m} \\
(176)\end{array}$ & $\begin{array}{c}D 400-700 \mathrm{~m} \\
(180)\end{array}$ & $\begin{array}{c}D 700-1300 \mathrm{~m} \\
(184)\end{array}$ & $\begin{array}{c}D>1300 \mathrm{~m} \\
(180)\end{array}$ \\
\hline$\# \mathrm{C}$ & 142 & 18 & 15 & 12 & 26 & 33 & 38 \\
$\# \mathrm{H}$ & 692 & 94 & 123 & 116 & 128 & 123 & 107 \\
$\# \mathrm{X}$ & 95 & 19 & 12 & 27 & 12 & 9 & 16 \\
\hline$C_{\text {bin }}$ & $13.1 \pm 1.1 \%$ & $9.9 \pm 2.3 \%$ & $8.3 \pm 2.2 \%$ & $6.8 \pm 2.0 \%$ & $14.4 \pm 2.8 \%$ & $17.9 \pm 3.1 \%$ & $21.1 \pm 3.4 \%$ \\
$S_{\text {bin }}$ & $63.9 \pm 2.4 \%$ & $51.9 \pm 5.4 \%$ & $68.3 \pm 6.2 \%$ & $65.9 \pm 6.1 \%$ & $71.1 \pm 6.3 \%$ & $66.8 \pm 6.0 \%$ & $59.4 \pm 5.7 \%$ \\
$X_{\text {bin }}$ & $8.8 \pm 1.0 \%$ & $10.5 \pm 2.4 \%$ & $6.7 \pm 1.9 \%$ & $15.3 \pm 3.0 \%$ & $6.7 \pm 1.9 \%$ & $4.9 \pm 1.6 \%$ & $8.9 \pm 2.2 \%$ \\
\hline
\end{tabular}

Table 6. Median orbital parameters for the taxonomic groups considered in our enlarged survey of NEOs.

\begin{tabular}{lcccccc}
\hline \hline & $a(\mathrm{au})$ & $e$ & $i(\mathrm{deg})$ & $q(\mathrm{au})$ & $Q(\mathrm{au})$ & MOID $(\mathrm{au})$ \\
\hline $\mathrm{A}$ & 1.642 & 0.441 & 6.706 & 1.001 & 2.398 & 0.034 \\
$\mathrm{C}$ & 1.775 & 0.452 & 8.199 & 0.989 & 2.570 & 0.066 \\
$\mathrm{D}$ & 1.771 & 0.414 & 8.158 & 0.999 & 2.536 & 0.051 \\
$\mathrm{~K}$ & 1.548 & 0.401 & 5.705 & 0.985 & 2.110 & 0.038 \\
$\mathrm{~L}$ & 1.272 & 0.393 & 8.123 & 0.926 & 2.522 & 0.037 \\
$\mathrm{~S}$ & 1.672 & 0.456 & 8.739 & 0.959 & 2.477 & 0.062 \\
$\mathrm{~V}$ & 1.625 & 0.438 & 9.770 & 0.910 & 2.349 & 0.045 \\
$\mathrm{X}$ & 1.595 & 0.423 & 8.718 & 0.949 & 2.053 & 0.060 \\
\hline
\end{tabular}

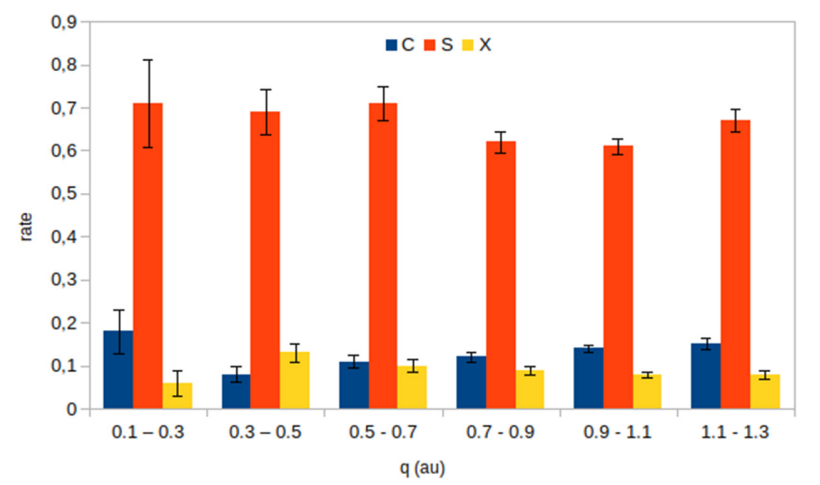

Fig. 2. Ratio of C-, S-, and X-complex bodies distributed according to their increasing perihelion. Error bars were computed using a standard Poisson approach.

predicted by observations. Granvik (2019) suggested the influence of thermal disruption as a possible explanation (i.e., objects passing closer to the Sun are disintegrated by a thermally driven mechanism), which should be more effective for darker carbonaceous bodies (Delbo et al. 2014). To confirm this hypothesis, we compared the ratio of $\mathrm{C}-$-, S-, and X-complex NEOs at different perihelion distances. As shown in Fig. 2, the ratio for $\mathrm{S}$ - and $\mathrm{X}$-complex NEOs are similar in each $q$ bin, and in general to the overall representation among the NEO population. The ratio for C-complex bodies instead decreases from 0.15 for bodies with the farthest passage $(1.1<q<1.3)$ to only 0.08 for those with $0.3<q<0.5$. They also represent almost $20 \%$ of the NEOs with the smallest perihelion. However, the large error bars associated with this scarcely populated bin (in our sample only 17 NEOs have $q<0.3 \mathrm{au}$ ) suggest that we should take the idea that the shortage of small perihelion C-type NEOs is thermally driven with precaution.
Table 7. Median orbital parameters sorted for estimated diameter considered in our enlarged survey.

\begin{tabular}{lcccccc}
\hline \hline & $a(\mathrm{au})$ & $e$ & $i(\mathrm{deg})$ & $q(\mathrm{au})$ & $Q(\mathrm{au})$ & MOID $(\mathrm{au})$ \\
\hline$D<60 \mathrm{~m}$ & 1.480 & 0.384 & 4.400 & 0.972 & 1.996 & 0.011 \\
$D 60-200 \mathrm{~m}$ & 1.382 & 0.395 & 6.922 & 0.945 & 1.869 & 0.034 \\
$D 200-400 \mathrm{~m}$ & 1.618 & 0.447 & 8.893 & 0.912 & 2.370 & 0.062 \\
$D 400-700 \mathrm{~m}$ & 1.701 & 0.452 & 9.427 & 0.969 & 2.387 & 0.078 \\
$D 700-1300 \mathrm{~m}$ & 1.830 & 0.477 & 12.590 & 0.961 & 2.685 & 0.100 \\
$D>1300 \mathrm{~m}$ & 2.145 & 0.533 & 14.292 & 1.034 & 3.237 & 0.150 \\
\hline
\end{tabular}

Finally, we analyzed the dependence of orbital parameters according to their estimated size retrieved in Sect. 4. The results shown in Table 7 indicate that smaller NEOs have lower median orbital parameters compared to larger ones. This is particularly evident for $e, i$, and MOID, which shows a steady increase in the average parameters. Interestingly, a positive correlation between diameter and semi-major axis $a$ could be indirect proof of the Yarkovsky effect, which is supposed to be more intense for smaller objects. There are two possible reasons why the Yarkovsky effect should act toward lowering the semi-major axis of our observed population. First, whilst the diurnal component can make a positive or negative change in $a$ according to the obliquity of the spin axis of the body, the observed excess of retrograde rotators within the NEO population related to the delivery mechanism from the most important NEO feeding resonances (La Spina et al. 2004) is prone to favor an overall decrease of the semi-major axis. Secondly, the seasonal effect, though smaller in strength, is proportional to the squared sine of the spin-axis obliquity, thus always resulting in a decrease in $a$ (Vokrouhlický et al. 2015). However, it is important to remember that observational biases can naturally lead to a decrease of the orbital parameter while considering smaller asteroids: for once, discovery surveys will miss most of the small bodies with large $a, e, i$ because of their relative motion on the sky during the fly-by. Moreover, small NEOs with higher inclination tend to pass rapidly onto the ecliptic plane, which makes them observable only for a limited amount of time; finally, objects with smaller $a$ have a shorter revolution periods, which means they have repeated passages close to Earth during which they can be discovered.

\subsection{Selection of potential targets of space missions}

A NEO with a MOID with the Earth $<0.05$ au and an absolute magnitude of $H<22$ is often defined as a potentially hazardous asteroid (PHA). These bodies pass close to our planet and they are usually big enough (at least $140 \mathrm{~m}$ ) to result in at least a 


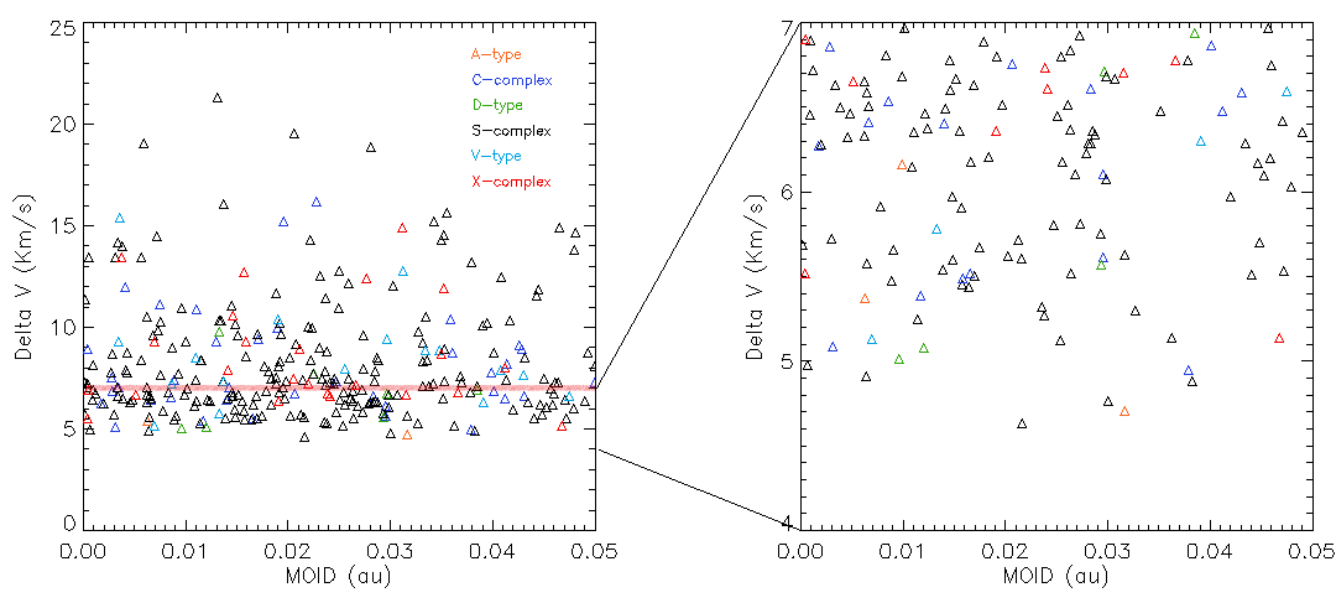

Fig. 3. MOID vs. $\Delta V$ for (left) all the PHAs considered in our extended survey and (right) limited to the accessible $\Delta V<7 \mathrm{~km} \mathrm{~s}^{-1}$. We identified 25 targets, shown in Table 8, that represent a better choice for next-generation space missions.

regional disruption event. Therefore, they represent all potential impactors on our planet. In our enlarged sample, we reclassified 314 PHAs: the overall majority (215 targets) belong to the S-complex group, while only 38 PHAs are classified as C-complex bodies.

A parameter often used to define the accessibility of a potential target of a space mission is the $\Delta V$, the velocity increment that must be applied to the spacecraft to reach the target's orbit. Current propulsion technologies limit the accessible rendez-vous NEOs to those with a $\Delta V<7 \mathrm{~km} \mathrm{~s}^{-1}$ (Hinkle et al. 2014). 179 PHAs characterized in our survey show a $\Delta V>7 \mathrm{~km} \mathrm{~s}^{-1}$, suggesting that they are virtually inaccessible by a hypothetical characterization mission. In Fig. 3, we reported the Earth MOID for these objects, together with their computed $\Delta \mathrm{V}$. Once again, the overall majority of these inaccessible PHAs belong to the S-complex (124), with only a few targets (21) showing a C-complex nature.

A low $\Delta V$ can also indicate that less fuel is needed onboard, and thus a shorter (and cheaper) mission (Ieva et al. 2014). In our survey, 135 PHAs have a $\Delta V<7 \mathrm{~km} \mathrm{~s}^{-1}$, meaning that they can all be potential targets of future space missions. The majority of these candidates belong to the S-complex. However, among the PHAs suitable to become future targets of space missions, we selected few bodies that could maximize the scientific return of a mission. We found $17 \mathrm{C}$-complex and five D-type objects, all bodies pertaining to taxonomic classes linked with a primitive composition and known to be extremely rare among the low $\Delta \mathrm{V}$ population (Kuroda et al. 2014). Incidentally, two of them have already been chosen as a sample-return candidate: (101955) Bennu, target of the OSIRIS-REx mission (Lauretta et al. 2017), and (175706) 1996 FG3, backup target of the ESA candidate mission MarcoPolo-R (Perna et al. 2013b). In addition, we also considered three A-type PHAs in this subgroup: a type of olivine-dominated asteroid extremely rare in both the NEO population and the asteroid belt (see also Popescu et al. 2018). These 25 bodies, reported in Table 8, due to their potential hazard, their peculiar spectral type, and their extreme low $\Delta \mathrm{V}$, represent a very good choice for the next generation of space missions towards an NEO.

\section{Discussion and conclusion}

With our extended survey, we classified 118 NEOs (Ieva et al. 2018 and this work) that had no data published in literature at the
Table 8. Potential targets of space missions, as discussed in the text.

\begin{tabular}{|c|c|c|}
\hline Object & $\begin{array}{c}\text { Taxonomy } \\
\text { complex/class }\end{array}$ & $\begin{array}{c}\Delta V \\
\left(\mathrm{~km} \mathrm{~s}^{-1}\right)\end{array}$ \\
\hline 1994 CJ1 & A-type & 4.704 \\
\hline$(451370)$ & C-complex & 4.948 \\
\hline 2011 AM24 & D-type & 5.017 \\
\hline 2009 DL46 & D-type & 5.080 \\
\hline (101955) & C-complex & 5.087 \\
\hline 2014 YS34 & A-type & 5.373 \\
\hline (440212) & C-complex & 5.389 \\
\hline 2011 BN24 & C-complex & 5.481 \\
\hline (388945) & C-complex & 5.520 \\
\hline (162998) & D-type & 5.568 \\
\hline 2016 AZ8 & C-complex & 5.614 \\
\hline (443880) & C-complex & 6.106 \\
\hline 2014HK129 & A-type & 6.155 \\
\hline (90416) & C-complex & 6.274 \\
\hline (65679) & C-complex & 6.405 \\
\hline (7753) & C-complex & 6.408 \\
\hline (281375) & C-complex & 6.477 \\
\hline (138175) & C-complex & 6.537 \\
\hline (4015) & C-complex & 6.587 \\
\hline (175706) & C-complex & 6.609 \\
\hline 2005 DD & D-type & 6.711 \\
\hline$(3671)$ & C-complex & 6.758 \\
\hline (269690) & C-complex & 6.859 \\
\hline (29075) & C-complex & 6.864 \\
\hline (289315) & D-type & 6.937 \\
\hline
\end{tabular}

moment of their observations. We confirmed that almost $60 \%$ of our NEO sample belongs to the S-complex, in agreement with literature (see Binzel et al. 2015). The majority of silicate NEOs is confirmed even when we considered a much larger sample, putting together data collected from Perna et al. (2018), Devogèle et al. (2019), and the MITHNEOS campaign (Binzel et al. 2019). Silicate bodies also dominate the PHA subgroup, a class of NEOs that deserve special attention due to their potential hazard to our planet. Among the 314 PHAs analyzed in our enlarged sample, most of them are at the moment inaccessible from Earth, and the overall majority of them belong to the S-complex. However, among the ones accessible for a characterization mission, 
we identified potential targets of future fly-by, rendez-vous, or even sample return missions. In particular, few primitive (C-and D-types) PHAs, due to their accessibility from Earth and their being of high scientific interest, represent extremely good candidates.

The dominance of silicate bodies among NEOs is probably related to the general high efficiency of transport mechanisms from the inner main belt (where silicate bodies are predominant, as confirmed by our orbital analysis) toward near-Earth orbits (Fieber-Beyer et al. 2012). Interestingly, we also found a positive correlation between the semi-major axis and diameter, which could be interpreted as indirect proof of the Yarkovsky effect. However, if we limit our analysis to objects with $H>24$, the relative percentage of silicates decreases from 64 to $44 \%$ with respect to the whole sample, while the contribution of lowalbedo material associated with a primitive composition (D-, $\mathrm{K}$ - and L-type NEOs) increases for larger $\mathrm{H}$, going from 7 to $21 \%$.

However, C-complex contribution in our analysis stay essentially fixed, close to the $\sim 15 \%$ suggested by optical surveys, the ones that more frequently discover new NEOs; at the same time infrared surveys, less biased toward fainter objects, suggest a total contribution of dark NEOs close to $\sim 30 \%$ (Mainzer et al. 2015). Our analysis found a larger percentage of dark and primitive-related bodies going toward larger $\mathrm{H}$, possibly suggesting that the missing pristine material in the NEO population could reside among fainter asteroids. This could also explain why these classes are extremely rare in the NEO population.

The dimensional analysis presented in this paper enlarges the previous sample of taxonomically classified NEOs shown in Ieva et al. (2018). Using a much larger sample (1081 NEOs), we confirmed that there is a lower contribution of carbonaceous bodies below $D<400 \mathrm{~m}$. This could be due to an observational bias that favors the discovery of larger darker asteroids and related to the fact that their lower average albedo (with respect, e.g., to silicate bodies) will eventually result in a larger estimated diameter.

To continue our characterization of NEOs and assess the number of primitive objects among the NEO population, we need to observe even fainter bodies in order to infer the real number of dark NEOs and constrain the importance of observational biases (with respect to an actual shortage of carbonaceous bodies due to a hypothetical limiting physical mechanism). These observations must be performed using larger telescopes (e.g., LBT) and/or at different wavelengths, in a range less biased than optical observations toward faint dark asteroids. The physical characterization of these very faint and small NEOs must also be timely, before they become too faint, or lost forever. Finally, new laboratory experiments performed on the samples returned from the Hayabusa2 and OSIRIS-REx mission will help unveil the role of porosity in the fragmentation of carbonaceous material.
Acknowledgements. This research has been funded with support from the European Commission (grant agreement no: 640351 H2020- PROTEC-2014 Access technologies and characterization for Near Earth Objects (NEOs). We also acknowledge financial support from ASI (contract No. 2017-37-H.0 CUP F82F17000630005: "OSIRIS-REx Partecipazione scientifica alla missione per la fase E”). D.P. has received funding from the European Union's Horizon 2020 research and innovation program under the Marie Sklodowska-Curie actions (grant agreement n. 664931). S.I. thanks F. Peluso for his fundamental contribution in the redaction of this paper. Part of the data utilized in this publication were obtained and made available by the MITHNEOS MIT-Hawaii Near-Earth Object Spectroscopic Survey. The IRTF is operated by the University of Hawaii under Cooperative Agreement no. NCC 5-538 with the National Aeronautics and Space Administration, Office of Space Science, Planetary Astronomy Program. The MIT component of this work is supported by NASA grant 09-NEOO0090001, and by the National Science Foundation under Grants Nos. 0506716 and 0907766. We finally thank the anonymous referee for her/his great help in improving this paper.

\section{References}

Binzel, R. P., Reddy, V., \& Dunn, T. L. 2015, Asteroid IV, eds. P. Michel, F. E. DeMeo, \& W. F. Bottke (Tucson, AZ: University of Arizona Press), 243 Binzel, R. P., DeMeo, F. E., Turtelboom, E. V., et al. 2019, Icarus, 324, 41 Burbine, T. H., Meibom, A., \& Binzel, R. P. 1996, Meteorit. Planet. Sci., 31, 607

Calla, P., Fries, D., \& Welch, C. 2018, ArXiv e-prints [arXiv:1808.05099] Cheng, A. F., Rivkin, A. S., Michel, P., et al. 2018, Planet. Space Sci., 157, 104 Delbo, M., Libourel, G., Wilkerson, J., et al. 2014, Nature, 508, 233

DeMeo, F. E., Binzel, R. P., Slivan, S. M., \& Bus, S. J. 2009, Icarus, 202, 160

Devogèle, M., Moskovitz, N., Thirouin, A., et al. 2019, AJ, 158, 196

Dotto, E., Della Corte, V., Amoroso, M., et al. 2020, BAAS, 52, 412.06

Fieber-Beyer, S. K., Gaffey, M. J., Hardersen, P. S., \& Reddy, V. 2012, Icarus, 221, 593

Granvik, M. 2019, in EPSC-DPS Joint Meeting 2019, EPSC-DPS2019-1739

Granvik, M., \& Brown, P. 2018, Icarus, 311, 271

Hinkle, M. L., Moskovitz, N., \& Trilling, D. 2014, AAS/Division Planet. Sci. Meeting Abstracts, 46, 213.07

Ieva, S., Dotto, E., Perna, D., et al. 2014, A\&A, 569, A59

Ieva, S., Dotto, E., Lazzaro, D., et al. 2016, MNRAS, 455, 2871

Ieva, S., Dotto, E., Mazzotta Epifani, E., et al. 2018, A\&A, 615, A127

Kuroda, D., Ishiguro, M., Takato, N., et al. 2014, PASJ, 66, 51

La Spina, A., Paolicchi, P., Kryszczyńska, A., \& Pravec, P. 2004, Nature, 428, 400

Lauretta, D. S., Balram-Knutson, S. S., Beshore, E., et al. 2017, Space Sci. Rev., 212, 925

Mainzer, A., Usui, F., \& Trilling, D. E. 2015, Space-Based Thermal Infrared Studies of Asteroids, eds. P. Michel, F. E. DeMeo, \& W. F. Bottke (Tucson, AZ: University of Arizona Press), 89

Mommert, M. 2017, Astron. Comput., 18, 47

Perna, D., Barucci, M. A., \& Fulchignoni, M. 2013a, A\&ARv, 21, 65

Perna, D., Dotto, E., Barucci, M. A., et al. 2013b, A\&A, 555, A62

Perna, D., Barucci, M. A., Fulchignoni, M., et al. 2018, Planet. Space Sci., 157, 82

Popescu, M., Perna, D., Barucci, M. A., et al. 2018, MNRAS, 477, 2786

Ryan, E. L., \& Woodward, C. E. 2010, AJ, 140, 933

Saito, J., Miyamoto, H., Nakamura, R., et al. 2006, Science, 312, 1341

Sanchez, J. A., Reddy, V., Kelley, M. S., et al. 2014, Icarus, 228, 288

Scheeres, D. J. 2018, Icarus, 304, 183

Veverka, J., Robinson, M., Thomas, P., et al. 2000, Science, 289, 2088

Vokrouhlický, D., Bottke, W. F., Chesley, S. R., Scheeres, D. J., \& Statler, T. S.

2015, Asteroids IV (Tucson, AZ: University of Arizona Press), 509

Watanabe, S.-i., Tsuda, Y., Yoshikawa, M., et al. 2017, Space Sci. Rev., 208, 3 